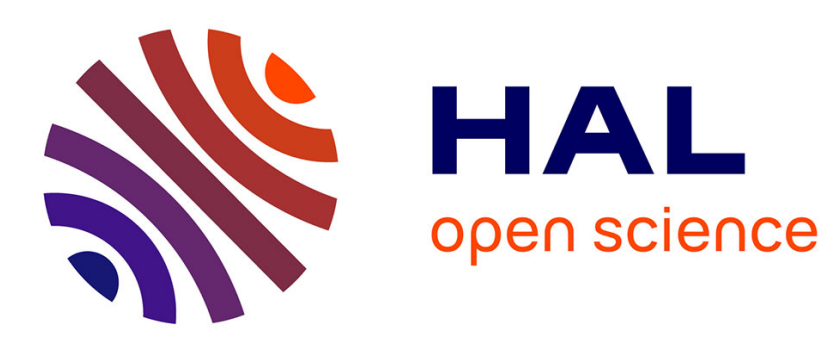

\title{
Spécificité des stratégies de coping des patients en rémission de la maladie de Crohn : une étude qualitative
}

\author{
A Lainé, A Laurent, S Nancey, B Flourié
}

\section{To cite this version:}

A Lainé, A Laurent, S Nancey, B Flourié. Spécificité des stratégies de coping des patients en rémission de la maladie de Crohn: une étude qualitative. Pratiques Psychologiques, A paraître, 10.1016/j.prps.2021.04.003 . hal-03545697

\section{HAL Id: hal-03545697 \\ https://hal.science/hal-03545697}

Submitted on 27 Jan 2022

HAL is a multi-disciplinary open access archive for the deposit and dissemination of scientific research documents, whether they are published or not. The documents may come from teaching and research institutions in France or abroad, or from public or private research centers.
L'archive ouverte pluridisciplinaire HAL, est destinée au dépôt et à la diffusion de documents scientifiques de niveau recherche, publiés ou non, émanant des établissements d'enseignement et de recherche français ou étrangers, des laboratoires publics ou privés. 


\title{
Spécificité des stratégies de coping des patients en rémission de la maladie de Crohn : une étude qualitative
}

\section{Specificity of coping strategies among patients with Crohn's disease in remission: A qualitative analysis}

\author{
A. Lainéa ${ }^{\mathrm{a}}$ A. Laurent ${ }^{\mathrm{b}, \mathrm{c}, *}, \mathrm{~S}$. Nancey ${ }^{\mathrm{d}}$, B. Flourié $^{\mathrm{d}}$ \\ a Laboratoire de psychologie (EA3188), université de Bourgogne Franche Comté, 25000 Besançon, France \\ b Laboratoire Psy-DREPI EA 7458, université Bourgogne Franche-Comté, 21000 Dijon, France \\ c Service d'anesthésie et de réanimation chirurgicale, CHU F. Mitterrand, 21000 Dijon, France \\ d Service de gastro-entérologie, CHU Lyon-Sud, Lyon, Lyon, France
}

\section{N F O A R T I C L E}

Historique de l'article :

Reçu le 27 novembre 2020

Accepté le 15 avril 2021

Disponible sur Internet le xxx

\section{Mots clés :}

Stratégies de coping

Maladie de Crohn

Savoir expérientiel

Émotions positives

Méthode qualitative

\begin{abstract}
R É S U M É
Introduction. - La maladie de Crohn (MC), maladie inflammatoire chronique de l'intestin, entraîne de fortes répercussions psychologiques et sociales en lien avec la spécificité de la symptomatologie. Pour mieux comprendre comment les patients font face à la maladie, les stratégies de coping ont été étudiées mais sans prendre en compte la spécificité du vécu de la MC.

Objectif. - Identifier les stratégies de coping perçues utilisées par les patients en rapport au vécu de leur maladie.

Méthode. - Trente-trois entretiens semi-directifs ont été menés auprès de patients MC en rémission et analysés via une analyse de contenu thématique.

Résultats. - Nos résultats mettent en évidence des stratégies de coping absentes des échelles de coping : l'expérience de la maladie paraît fondamentale dans l'établissement de nouvelles stratégies centrées sur des savoirs expérientiels utilisés afin de réduire le stress induit par la survenue potentielle d'une rechute. Des stratégies de coping basées sur des émotions positives à partir desquelles des stratégies de mises en sens de la maladie peuvent également être mises en place.
\end{abstract}

\footnotetext{
* Auteur correspondant. Laboratoire Psy-DREPI, Esplanade Erasme, university of Bourgogne Franche-comté, 21000 Dijon, France.

Adresse e-mail : Alexandra.laurent@u-bourgogne.fr (A. Laurent).
} 
Conclusion. - Les stratégies de coping "savoir expérientiel " et " émotions positives " peuvent permettre de mieux comprendre la complexité du vécu de la MC et orienter l'accompagnement psychologique des patients.
Keywords:

Coping strategies

Crohn's disease

Experiential knowledge

Positive emotions

Qualitative approach

\section{A B S T R A C T}

Introduction. - Crohn's disease (CD), a chronic inflammatory bowel disease, has strong psychological and social repercussions related to the specificity of the symptomatology. To better understand how patients cope with the disease, coping strategies have been studied but without taking into account the specificity of the $\mathrm{CD}$ experience. Objective. - Our objective is to identify the perceived coping strategies used by patients in relation to their illness experience. Method. - Using a qualitative approach, semi-structured interviews and thematic content analysis with $33 \mathrm{CD}$ patients in remission were conducted.

Results. - Our results highlight that some of the coping strategies used are not taken into account by the coping scales frequently used in the literature. Indeed, the illness experience appears to be fundamental in the establishment of new strategies based on the experiential knowledge patients use to reduce the stress induced by a potential relapse. Moreover, coping strategies based on positive emotions are also implemented, and they enable patients to make sense of the disease.

Conclusion. - The coping strategies, i.e., "experiential knowledge" and "positive emotions", may shed more light on the complexity of the illness experience of $\mathrm{CD}$ patients and allow us to make recommendations concerning the psychological support of patients.

\section{Introduction}

La maladie de Crohn (MC) est considérée avec la rectocolite hémorragique (RH) comme une maladie inflammatoire chronique de l'intestin (MICI). Les symptômes sont principalement des douleurs abdominales, des diarrhées, de la fatigue, une perte de poids, et dans les cas les plus sévères, des abcès ou des fistules. Évoluant par poussées, on estime le risque de rechute de la maladie entre $30 \%$ et $60 \%$ par année (Van der Have et al., 2013). À ce jour, l'étiologie de la rechute reste inconnue sur le plan biologique et psychologique. Face à cette méconnaissance des facteurs de rechute, la qualité de vie des patients est fortement altérée (Van der Have et al., 2013). Dans ce sens, une récente revue de la littérature conduite par Byron et al., 2020 portant sur les expériences des patients de leur MICI met en évidence l'apparition de troubles émotionnels chez ces patients. Elle souligne également la difficulté pour ceux-ci de composer avec la dimension imprévisible de la maladie ainsi que les nombreux efforts fournis par ceux-ci pour maintenir une vie normale. En effet, il semble se dégager une spécificité du vécu de la maladie découlant des modifications corporelles et des répercussions symptomatologiques (Casati, Toner, De Rooy, Drossman, \& Maunder, 2000 ; Irvine, 2004).

Ainsi, on rencontre fréquemment chez les patients atteints de la MC, un effondrement dépressif en lien avec l'incapacité pour les patients à maintenir leur profession dû aux symptômes de la maladie. Cette perte de statut de travailleur actif est vécue comme une fragilisation des processus identitaires, traduite par un sentiment d'auto dévalorisation et une incapacité à se maintenir dans les 
rôles familiaux et sociaux établis auparavant (Aïni, Curelli-Chéreau, \& Antoine, 2010). En parallèle, les patients ressentent un sentiment d'injustice exacerbé par la méconnaissance de l'étiologie de la maladie (McCormick et al., 2012). Les patients rapportent également un sentiment de honte qui trouve sa source dans les symptômes gastro-intestinaux, comme les diarrhées ou les flatulences. Ils ne sentent ni compris, ni écoutés au niveau social et tendent à se dévaloriser sur le plan narcissique (Cooper, Collier, James, \& Hawkey, 2010). Les patients sont en demande de réussir à trouver des moyens leur permettant de vivre comme tout le monde. Dans ce sens, ils évoquent une peur de la stigmatisation vécue dans la sphère familiale et conjugale. L'étude de Taft, Keefer, Artz, Bratten, et Jones (2011) montre que cette peur entraînerait une diminution de l'estime de soi ainsi qu'une augmentation de la détresse psychologique.

Afin de mieux comprendre comment les patients arrivent à faire face à un tel vécu, de nombreux auteurs ont centré leurs travaux sur les stratégies de coping. Définies comme «l'ensemble des efforts cognitifs et comportementaux destinés à maîtriser, réduire ou tolérer les exigences internes ou externes qui menacent ou dépassent les ressources d'un individu " (Lazarus and Folkman, 1991, p141) elles représentent un enjeu majeur dans la prise en charge des patients. En effet, plusieurs études ont montré un lien entre stratégies de coping et évolution de la maladie (Bitton et al., 2008 ; Pelissier, Dantzer, Canini, Mathieu, \& Bonaz 2010 ; Lainé et al., 2016). Elles paraissent également être déterminantes dans le niveau de qualité de vie des patients (Maes, Leventhal, \& De Ridder, 1996 ; Stroebe, Shut, \& Stroebe, 1995 ; Luo et al., 2018). Toutefois, les nombreux travaux menés dans ce domaine évaluent les stratégies de coping en s'appuyant sur des outils génériques comme le Ways of Coping Checklist (Vitaliano, Russo, Carr, \& Maiuro, 1985), le Brief Cope (Carver, 1997), le Coping Inventory for Stressful Situations (Endler \& Parker, 1990) ou le COPE Inventory (Carver, Scheier, \& Weintraub, 1989), utilisés pour mesurer l'adaptation à divers stresseurs rencontrés aussi bien dans le cadre de la maladie, que dans la sphère sociale ou professionnelle. Même s'il a pu être montré un effet délétère des stratégies de coping évitantes ou centrées sur l'émotion des patients atteints de maladies chroniques (Mc Combie, Mulder, \& Gearry, 2013), il est important de souligner que les stratégies mesurées à travers ces outils restent limitées à celles classiquement retrouvées dans la population générale et donc discutable au niveau de population vivant des situations spécifiques telles que la maladie (Livneh, 2019). En effet, très peu d'études qualitatives ont permis de mettre en lumière les stratégies déployées par les patients pour faire face à leur MICI (Byron, Cornally, Burton, Savage, 2020). Pourtant, les auteurs Larsson, Lööf, et Nordin (2017) soulignent que les patients atteints de MICI sont enclins à utiliser des stratégies comportementales et émotionnelles spécifiques au stress associé à la maladie.

L'objectif de notre étude est de poursuivre cette perspective d'évaluation qualitative des stratégies de coping, en partant du vécu passé et actuel de la maladie par les patients atteints de MC. Il s'agit d'identifier les stratégies de coping des patients centrées sur le problème, l'émotion et la recherche de soutien social et faire apparaître d'autres stratégies d'ajustement fondées sur leur expérience de la MC.

\section{Méthode}

\subsection{Participants}

Cette recherche est une étude ancillaire d'un projet hospitalier de recherche clinique conduit par le service de gastroentérologie de Lyon Sud et portant sur les marqueurs immunologiques, inflammatoires et psychologiques de la rechute de la MC auprès de 140 participants. Celle-ci a reçu un avis favorable du CPP Sud Est II.

Trente-trois patients ( 21 femmes, 12 hommes, d'âge moyen 39,12 (écart-type 12,98) atteints de la MC depuis en moyenne 9 ans (écart-type 6,77) ont été sélectionnés au fur et à mesure de l'inclusion des patients pour la recherche initiale (Tableau 1). Les sujets éligibles étaient en rémission au moment de leur inclusion, âgés de plus de 18 ans et sans traitements médicamenteux. L'âge moyen auquel la maladie a été diagnostiquée est de 29 ans (écart-type 13,51). L'équipe médicale prenant en charge les patients aux Hôpitaux Lyon Sud, a évalué en amont la fréquence des rechutes, la durée de la maladie et la présence d'antécédent de résection afin que l'ensemble de ces caractéristiques puissent être 
Tableau 1

Caractéristique de l'échantillon.

\begin{tabular}{lrc}
\hline & $n=33(\%)$ & Moyenne (écart-type) \\
\hline Variables sociodémographiques & & $39(12,8)$ \\
Âge (y), moyenne (écart-type) & & \\
Sexe & $12(36 \%)$ & \\
$\quad$ Homme & $21(64 \%)$ & \\
Femme & & $9(6,77)$ \\
Variables médicales & $12(36 \%)$ & \\
Fréquence des rechutes & $14(42 \%)$ & \\
$\quad$ Faible & $7(22 \%)$ & $29(13,51)$ \\
Modérée & & \\
$\quad$ Élevée & $10(30 \%)$ & \\
Durée de la maladie (y) & $23(70 \%)$ & \\
Antécédent de résection & & \\
Présence & & \\
Absence & & \\
Âge au diagnostic $(y)$ &
\end{tabular}

représentées dans l'échantillon de patients (Tableau 2). Avant de débuter les interviews, les patients ont été informés des objectifs de l'étude et ont signé un consentement éclairé.

\subsection{Récolte des données}

Des entretiens semi-directifs ont été conduits par une chercheuse en psychologie (AgL) avec pour objectif de porter notre attention sur le récit subjectif de la maladie. Ces entretiens ont été conduits de janvier à décembre 2015. Ils ont été enregistrés et intégralement retranscrits. Leur durée moyenne était de 57 minutes (écart-type 18,49). Au cours de ceux-ci, nous avons investigué le vécu passé et actuel de la maladie ainsi que les ressources mobilisées pour faire face aux stresseurs découlant de la MC (Tableau 3). L'inclusion des patients dans la recherche a été effective jusqu'à saturation des données (Bloor \& Wood, 2006), c'est-à-dire, jusqu'à ce qu'aucune nouvelle information ou thématique ne soit identifiée au sein des données, permettant une stabilisation de notre grille d'analyse thématique et l'absence de nouvelles catégorisations de coping (Guest, Bunce, \& Johnson, 2006 ; Francis et al., 2010).

\subsection{Analyse des données}

La méthode retenue pour l'analyse des données est celle de l'analyse thématique développée par Braun et Clarke (2006) dans une perspective « top down » ou déductive c'est-à-dire guidée au niveau théorique. Cette méthode nous permet de capturer des éléments signifiants sur les données par rapport à la question de recherche et de les structurer selon 6 phases : familiarisation avec les données, générer les grandes thématiques en lien avec les questions de recherche, rechercher les thèmes, réviser l'ensemble des thèmes et rédiger. L'analyse des entretiens a été effectuée par deux chercheuses en psychologie (Ag L, Al L) familiarisées à la méthode d'analyse qualitative (Laurent et al. 2019, Laurent et al. 2017). Un ensemble de thèmes a été identifié notamment sur le vécu de la maladie et de la rechute, toutefois dans le cadre des objectifs de cet article, nous porterons notre attention sur les thèmes relatifs aux stratégies de coping. Ces stratégies de coping ont été répertoriées puis classées en nous appuyant sur les données de la littérature en fonction de trois grandes stratégies de coping centrées sur le problème, l'émotion ou la recherche de soutien social (Lazarus \& Folkman, 1984 ; Carver et al., 1989), à l'aide du logiciel Nvivo10. Le codage a été réalisé en double aveugle. Des réunions régulières entre les chercheuses ont eu lieu afin de rendre compte des stratégies de coping identifiées et de trouver un accord inter-juges. Les stratégies de coping ont ensuite été présentées sous forme de tableau avec une définition pour chacune des stratégies et des illustrations de verbatims. 


\section{Tableau 2}

Caractéristiques des patients.

\begin{tabular}{|c|c|c|c|c|c|}
\hline Pseudonyme & Sexe & Âge (année) & Fréquence de rechute & Durée de la maladie (année) & Antécédents de résection \\
\hline Aurélie & $\mathrm{F}$ & 29 & Importante & 1 & Absence \\
\hline Fatima & $\mathrm{F}$ & 53 & Basse & 1 & Absence \\
\hline Audrey & $\mathrm{F}$ & 23 & Modérée & 4 & Absence \\
\hline Caroline & $\mathrm{F}$ & 31 & Importante & 16 & Absence \\
\hline Jacqueline & $\mathrm{F}$ & 74 & Basse & 4 & Présence \\
\hline Brigitte & $\mathrm{F}$ & 53 & Modérée & 18 & Absence \\
\hline Florence & $\mathrm{F}$ & 39 & Modérée & 4 & Absence \\
\hline Sophia & $\mathrm{F}$ & 35 & Modérée & 16 & Présence \\
\hline Vanessa & $\mathrm{F}$ & 36 & Modérée & 2 & Absence \\
\hline Martine & $\mathrm{F}$ & 58 & Basse & 5 & Présence \\
\hline Julie & $\mathrm{F}$ & 27 & Modérée & 6 & Absence \\
\hline Sandrine & $\mathrm{F}$ & 40 & Importante & 10 & Absence \\
\hline Thomas & $\mathrm{H}$ & 24 & Importante & 10 & Absence \\
\hline Bachir & $\mathrm{H}$ & 20 & Modérée & 2 & Absence \\
\hline Christophe & $\mathrm{H}$ & 39 & Basse & 15 & Présence \\
\hline Patrice & $\mathrm{H}$ & 41 & Basse & 7 & Absence \\
\hline Philippe & $\mathrm{H}$ & 45 & Modérée & 15 & Présence \\
\hline Monique & $\mathrm{H}$ & 66 & Basse & 21 & Absence \\
\hline Nicolas & $\mathrm{H}$ & 27 & Basse & 9 & Absence \\
\hline Alexis & $\mathrm{H}$ & 18 & Importante & 3 & Absence \\
\hline Redha & $\mathrm{H}$ & 33 & Modérée & 4 & Absence \\
\hline Sylvie & $\mathrm{F}$ & 48 & Modérée & 7 & Présence \\
\hline Valérie & $\mathrm{F}$ & 41 & Basse & 7 & Présence \\
\hline Nathalie & $\mathrm{F}$ & 42 & Basse & 5 & Absence \\
\hline Céline & $\mathrm{F}$ & 35 & Importante & 22 & Absence \\
\hline Corinne & $\mathrm{F}$ & 45 & Modérée & 22 & Absence \\
\hline Pascale & F & 49 & Basse & 7 & Absence \\
\hline Séverine & $\mathrm{F}$ & 38 & Basse & 5 & Présence \\
\hline Léa & $\mathrm{F}$ & 31 & Modérée & 16 & Absence \\
\hline Camille & $\mathrm{F}$ & 21 & Importante & 2 & Absence \\
\hline Stéphane & $\mathrm{H}$ & 52 & Modérée & 14 & Absence \\
\hline Nassim & $\mathrm{H}$ & 34 & Modérée & 22 & Présence \\
\hline Bruno & $\mathrm{H}$ & 44 & Basse & 13 & Présence \\
\hline
\end{tabular}

\section{Tableau 3}

Canevas d'entretiens.

Pouvez-vous nous raconter l'histoire de votre maladie ?

Aujourd'hui comment vivez-vous la maladie?

De quelle manière faites-vous face à la maladie ?

\section{Résultats}

L'analyse des données nous a permis d'identifier 17 stratégies de coping qui peuvent être répertoriées selon les trois grands types de coping référencés au sein de la littérature (Mc Combie et al., 2013) : centré sur le problème représenté par la gestion des symptômes et des rechutes (Tableau 4), centré sur les émotions associées à la maladie (Tableau 5) et enfin sur la recherche de soutien social (Tableau 6).

\subsection{Stratégies d'ajustement centrées sur le problème en lien avec la maladie de Crohn}

Le coping centré sur le problème désigne l'ensemble des efforts cognitifs et comportementaux d'un sujet pour modifier sa situation. Plusieurs stratégies sont mobilisées par les patients pour faire face à la maladie et prévenir la rechute. Les patients restent en état de vigilance quant à l'apparition de premiers symptômes en sondant leurs éprouvés corporels (vigilance face à la rechute). Ils planifient leurs activités et leurs déplacements futurs afin d'anticiper la proximité de toilettes ou de leur domicile en cas de survenue de symptômes (planification d'actions pour prévenir la rechute). Des stratégies de recherche 


\section{A. Lainé et al.}

\section{Tableau 4}

Les thèmes principaux et les sous-thèmes concernant les stratégies de coping centrées sur le problème.

\begin{tabular}{ll}
\hline Thèmes principaux & Sous thèmes \\
\hline Coping centré sur le problème & Vigilance face à la rechute \\
" Mais quand même j'ai bien intégré que quand même j'ai un risque d'un événement \\
exceptionnel grave et donc euh je suis très à l'écoute plutôt des douleurs. » Monique, 66 ans \\
Planification d'action pour prévenir la rechute \\
"J'essaie de tout prévenir au maximum ! On ne peut pas tout prévenir, tout contrôler, mais \\
maintenant on va dire qu'on sait que voilà y'a un risque. Je m'exige beaucoup de choses. Je \\
m'entoure de sécurité » Vanessa, 36 ans \\
Recherche d'informations sur la maladie \\
"J'essaie plutôt de capter ce que les gens vivent, ce que les gens ressentent pour me \\
comparer et moi me construire ma propre image ou voilà me situer par rapport à eux. " \\
Nicolas, 27 ans \\
Combattre la maladie \\
"Je me suis battu on va dire. Battu entre guillemets parce qu'il y a des choses beaucoup \\
plus graves. Mais bon c'est un p'tit combat on va dire, de tous les jours. " Patrice, 41 ans \\
S'appuyer sur un savoir expérientiel de la maladie \\
"Alors que maintenant je sais que quand j'ai des grosses douleurs et que je suis constipée il \\
faut mettre un lavement, ça aide. Ça enlève pas complètement la douleur, mais ça vous \\
aide à pas trop souffrir quand même, à essayer d'évacuer » Florence, 39 ans
\end{tabular}

Tableau 5

Les thèmes principaux et les sous-thèmes concernant les stratégies de coping centrées sur l'émotion.

\begin{tabular}{|c|c|}
\hline Thèmes principaux & Sous-thèmes \\
\hline Coping centré sur l'émotion & $\begin{array}{l}\text { Maîtriser ses émotions } \\
\text { "Comme on dit je me mets des coups de pieds au derrière et je me booste en me disant oui } \\
\text { il faut que tu ailles de l'avant ». Pascale, } 49 \text { ans } \\
\text { Relativiser sur l'apparition et la manifestation des symptômes } \\
\text { "Je sais que pour certaines personnes atteintes de la maladie de Crohn, c'est l'enfer quoi, } \\
\text { mais moi j'ai pas de diarrhée, j'ai pas de problème... La fistule, c'est mimine par rapport à } \\
\text { ce que ça pourrait être quoi. » Sandrine, } 40 \text { ans } \\
\text { Optimisme sur l'évolution de la maladie } \\
\text { "J'ai pas le sentiment qu'il faille s'en faire ». Monique, } 66 \text { ans } \\
\text { Eviter de penser à la maladie } \\
\text { "Je dirais que moins j'y pense et mieux ça va. Je veux être dans le présent et pas dans la } \\
\text { projection. " Aurélie, } 29 \text { ans } \\
\text { Usage de toxiques } \\
\text { "Je suis passée au cannabis pour m'aérer un petit peu la tête. » Sophia, } 35 \text { ans } \\
\text { Auto- accusation sur leur prise en charge de la maladie } \\
\text { "Je prends peut-être trop ça à la légère je ne sais pas. " Audrey, } 23 \text { ans } \\
\text { Perte de contrôle somatique et psychique } \\
\text { J'ai un tempérament assez têtue donc du coup je persiste toujours avec des. . il faut que je } \\
\text { le fasse ou il faut y aller. Des fois ça passe, des fois ça casse parce qu'en fait on est rattrapé } \\
\text { par son corps. Le corps ne veut plus alors que la tête si ». Léa, } 31 \text { ans } \\
\text { Fatalisme } \\
\text { "Oh pour moi oui un petit peu, je me dis qu'un jour je finirais peut-être par avoir un } \\
\text { cancer, mais bon qu'est-ce que vous voulez... » Jacqueline, } 74 \text { ans } \\
\text { Coping religieux } \\
\text { "Je pense que dieu il m'aide, je me rapproche de lui et puis il m'aide c'est tout. » Patrice, } \\
41 \text { ans } \\
\text { S'autoriser à prendre du plaisir } \\
\text { "Demain je serais peut-être sur un lit d'hôpital et je pourrais profiter de rien donc euh ben } \\
\text { tout l'argent que j'ai-je le dépense en me disant ben si au moins demain t'es couché ben } \\
\text { tout ce que j'aurais voulu faire je l'aurais fait. » Caroline, } 31 \text { ans } \\
\text { Ré-évaluation positive } \\
\text { "Donc il y a un côté positif peut-être que cette maladie me permet de mieux tolérer le } \\
\text { stress, ou en tout cas de le minimiser sur des situations qui ne le méritent pas. » Stéphane, } \\
52 \text { ans }\end{array}$ \\
\hline
\end{tabular}


Tableau 6

Thème principal et sous thème concernant les stratégies de coping centrées sur le soutien social.

Coping centré sur la recherche de Recherche de soutien informatif

soutien social

"J'ai envie d'aller vers les autres et d'en discuter, mais je n'ai pas la force d'aller les voir. Je me dis aller sur le site des associations, aller à ces réunions justement où vont les malades des MICl et voir leurs ressentis justement, comment ils vivent? Dans ma tête je ne sais pas il me semble que ça pourrait m'aider. " Nassim, 34 ans

Recherche de soutien familial et social

"J'arrivais pas à en parler, c'est pas possible, parce que je ne voulais pas me sentir à l'écart, qu'on dise oh la pauvre. Le seul à qui je peux en parler sans tabou c'est mon mari. . il me soutient et ça m'aide ». Sophia, 36 ans

d'informations portent sur les différentes formes que peut revêtir la MC. Internet et les autres patients représentent les principales sources d'informations sur la symptomatologie et le vécu des troubles (recherche d'information sur la maladie). Ils décrivent un combat, une lutte contre une maladie perçue comme invisible puisque les symptômes portent sur l'intérieur du corps, et imprévisible dans la mesure où les facteurs de risque de rechute ne sont pas connus (combattre la maladie).

À partir de leurs expériences de la maladie acquises avec le temps, les patients ont obtenu des connaissances sur la manifestation des symptômes et ont pu tester l'efficacité de certaines stratégies personnelles comme des restrictions alimentaires ou la pratique de lavements pour réduire les douleurs abdominales et prévenir la rechute (s'appuyer sur un savoir expérientiel de la maladie). Leurs expériences leur offrent un sentiment de réassurance et la capacité de trouver des solutions. Celles-ci ont pour effet de les responsabiliser vis-à-vis de leur état de bien-être.

\subsection{Stratégies de coping centrées sur les émotions ressenties en lien avec la maladie de Crohn}

Les stratégies de coping centrées sur l'émotion indiquent les efforts visant à supporter ou atténuer les émotions induites par la maladie. Plusieurs patients s'appuient sur l'idée d'une force de caractère pour affronter le stress induit par la maladie. Certains décrivent leur capacité à se remobiliser face à la lassitude de combattre la pathologie. Convaincus que le stress peut précipiter la rechute, ils tentent de maîtriser leurs émotions négatives lors de situations professionnelles et/ou familiales stressantes (maîtriser ses émotions). Les sujets perçoivent leur maladie comme moins sévère que celle d'autres malades chroniques. Ils relativisent sur l'âge d'apparition de la maladie ainsi que sur le caractère passager des rechutes (relativiser sur l'apparition et la manifestation des symptômes). Ils déclarent rester positifs sur l'évolution de la maladie, une longue période de rémission ou l'amélioration de leur état de santé quitte parfois à baser leur raisonnement sur des superstitions ou des pensées magiques (optimisme sur l'évolution de la maladie).

Des stratégies d'évitement sont présentes. Certains sujets préfèrent ne pas connaître les différentes formes évolutives de la maladie. Ils s'abstiennent de poser des questions à leurs médecins ou de fréquenter les associations de patients. Le fait d'éviter de se concentrer sur la maladie semble diminuer leur anxiété face à une potentielle récidive et augmenter leur état de bien être subjectif. Il convient donc de multiplier les sources de distractions et d'occupations (éviter de penser à la maladie). Ces stratégies d'évitement peuvent être soutenues par une consommation de substances toxiques destinées à les apaiser (usage de toxiques). Certains patients expriment avec culpabilité, le sentiment de ne pas prendre suffisamment au sérieux le fait d'être malade ou de ne pas être assez compliants aux médecins (auto-accusations sur leur prise en charge de la maladie). Parallèlement, certains patients endossent un statut de victime en déclarant ne pas avoir le choix sur la gestion de la maladie. Proche de la résignation, leur avenir leur apparaît comme écrit à l'avance (fatalisme). Devant l'imprévisibilité des symptômes, les sujets font l'expérience d'un défaut de maîtrise de leur propre corps avec l'impossible rétention des matières fécales, ou la survenue brutale de douleurs abdominales. Cette perte de contrôle somatique entraîne une perte de contrôle au niveau psychique. Corps et esprit semblent alors entrer en confrontation et fonctionner de manière dissociée (perte de contrôle somatique et psychique). Le recours à la spiritualité devient une source de courage et de force pour surmonter les épreuves induites par les symptômes de la pathologie (coping religieux). 
D’autres stratégies centrées sur les émotions positives ont été mises en évidence à travers le discours des patients. En effet, la survenue d'une potentielle rechute, les entraînent à valoriser le temps présent en profitant de chaque instant. Cette recherche de plaisir déculpabilisée et décomplexée fait écho aux souffrances antérieures vécues. Le plaisir apparaît comme un droit face à leurs souffrances (s'autoriser à se faire plaisir). De plus, les sujets effectuent un travail de mise en sens des fonctions de la maladie ou des rechutes. La MC est investie comme une figure externe bienveillante omniprésente qui offre au patient un état des lieux de leurs ressentis somatiques et psychiques en les obligeant à réagir en se protégeant. À ce sujet, ils décrivent une meilleure compétence à gérer les facteurs de stress externes à la maladie, pouvant à ce titre les prémunir contre un effondrement psychique et somatique. De plus, certains patients insistent sur la nécessité de profiter de chaque instant devant le risque d'apparition d'une potentielle rechute, ainsi que sur leur volonté de se centrer sur des plaisirs quotidiens ou d'affirmer leurs désirs (ré-évaluation positive).

\subsection{Stratégies centrées sur la recherche de soutien social}

Considérées comme les efforts visant à obtenir le soutien et l'aide d'autrui, les stratégies de coping centrées sur la recherche de soutien social sont peu mobilisées par les patients.

Si beaucoup de patients évoquent le soutien de leur entourage familial et médical, il semble qu'ils recherchent un soutien informatif apporté par les patients atteints de MC. Ce sont souvent les associations ou les réseaux sociaux qui constituent un relai pour trouver des espaces de dialogue relatifs aux traitements, à la gestion des symptômes et aux stratégies visant à prévenir l'évolution de la maladie. Cette démarche relève soit d'une simple curiosité soit d'un réel besoin d'étayage afin de limiter le stress perçu par la MC. Les patients mentionnent également que cette démarche reste difficile à entreprendre.

\section{Discussion}

À partir du discours des patients, l'objectif de notre étude était de mettre en lumière des stratégies de coping employées pour s'ajuster au vécu de la MC. Nos résultats permettent d'identifier et de caractériser des stratégies de coping communément décrites dans la littérature sur la MC : des stratégies de planification, d'anticipation et de recherche d'informations sur la maladie et les rechutes. Ces stratégies offrent aux patients un sentiment de maîtrise en écho à l'impossibilité de prédire les rechutes de la MC. Elles rendent la maladie omniprésente à l'esprit des sujets qui peuvent avoir un sentiment de perte de liberté. Pour autant elles semblent signer un refus de se résigner au statut de malade et permettent aux sujets d'accéder à de nouveaux remaniements identitaires, en relançant le travail d'acceptation de la MC. Nos résultats confirment également l'usage de stratégies d'évitement, et de minimisation pour tenter d'éviter une confrontation au réel de la MC (Graff et al., 2009 ; Larsson et al., 2017). L'ajustement à la maladie vient bouleverser l'équilibre psychique des sujets qui peuvent avoir l'impression d'un sentiment de perte de contrôle, entraînant des conduites d'auto-accusation et un fatalisme. Ces stratégies centrées sur des émotions négatives peuvent avoir un effet délétère pour la santé des patients qui se montreraient moins observants dans les prescriptions médicales (Maes et al., 1996).

L'importance de notre étude réside également dans le fait que l'approche qualitative des stratégies de coping met en avant l'existence de nouvelles stratégies qui ne sont pas étudiées dans les recherches basées sur une méthodologie quantitative.

\subsection{Le savoir expérientiel de la maladie de Crohn}

Dans un contexte de manque de connaissances médicales de la MC, les patients valorisent le fait de s'appuyer sur leurs propres expériences pour gérer les stresseurs induits par la maladie et une potentielle rechute. Leur expérience de la MC devient source de production de savoirs et de connaissances qui constituent des leviers sur lesquels ils peuvent s'appuyer pour transformer leur environnement et accroître leur survivabilité dans le contexte de la maladie chronique. Ainsi, des stratégies de planification d'action visant à prévenir la rechute sont spécifiques à certains patients. S'appuyer sur un 
savoir expérientiel de la MC apparaît alors comme une stratégie de coping proactive (Folkman, 2008) puisqu'elle prend en compte les événements passés et futurs en lien avec le vécu de la maladie. En rapport à la spécificité de la MC et aux travaux développés par Schwarzer et Renner (2000), la dimension temporelle paraît fondamentale dans la construction d'un sentiment d'auto-efficacité et la mise en place de coping centrées sur la maladie et la rechute. C'est via la construction de ressources personnelles fondées sur leurs expériences que les patients peuvent réaliser un travail de feedback sur leurs efforts passés afin de réajuster leurs actions présentes et futures. Les patients sont d'ailleurs plus actifs dans leur prise en charge et peuvent progressivement se responsabiliser, s'émanciper du pouvoir médical pour arriver à être autonomes.

Le savoir expérientiel se retrouve également au sein des stratégies de coping centrées sur la recherche de soutien social. En effet, la recherche de soutien ne porte pas sur l'entourage social ou familial du patient mais sur les autres malades de Crohn. Cette recherche semble porter sur une demande de soutien informatif, sous tendu par la question " mais comment font les autres pour gérer la maladie ? ". Cette stratégie apparaît alors comme une tentative de réduction du stress portant sur le risque de rechute ou d'aggravation de la maladie. Elle paraît également avoir pour visée un soutien émotionnel. La MC étant invisible, nombre de patients déclarent vivre dans le secret, se sentant incompris par leur famille, s'enfermant dans la honte des symptômes et souffrant du manque de sensibilisation du public pour cette maladie (Fourie, Jackson, \& Aveyard, 2018). En lien avec l'étude conduite par Hall, Rubin, Dougall, Hungin, et Neely (2005) qui retrouve des résultats similaires à notre étude, nous pouvons faire l'hypothèse que cette recherche d'ouverture sur le vécu de la maladie des autres patients témoigne de la nécessité de pouvoir s'identifier et se référer à eux sans être jugés.

\subsection{Fonction adaptative des émotions positives}

Nos résultats suggèrent que des stratégies de coping centrées sur des émotions négatives coexistent avec des stratégies de coping centrées sur des émotions positives. Ce phénomène décrit dans notre population est retrouvé chez plusieurs patients atteints de maladies chroniques (Folkman, Chesnay, Collette, Boccellari, \& Cooke, 1996 ; De Ridder, Geenen, Kuijer, \& Van Middendorp, 2008). Les difficultés induites par la maladie MC donnent lieu à des bénéfices secondaires pour les patients comme le droit pour les patients de multiplier les sources de plaisir, de prendre soin d'eux et d'affirmer leurs désirs vis-à-vis de leur entourage. Ainsi nous retrouvons des stratégies centrées sur la réévaluation positive de la maladie, l'optimisme et des stratégies compensatrices centrées sur la recherche de plaisir. Schwarzer et Knoll (2002) témoignent de l'effet bénéfique du coping positif. Les stratégies de coping centrées sur les émotions positives retrouvées dans notre étude semblent agir comme des fonctions adaptatives dans un contexte de stress induit par l'impossibilité à pouvoir prédire les rechutes et par le caractère stigmatisant des symptômes. Elles permettent aux patients de développer de nouvelles compétences émotionnelles agissant comme un support de revalorisation narcissique.

La saturation des données nous permet d'explorer de manière la plus exhaustive possible les différentes stratégies de faire face des patients atteints de MC. Nous avons rencontré uniquement des patients en rémission, il conviendrait de multiplier les entretiens en intégrant des patients en phase de rechute afin de mettre en lumière des stratégies de coping pour faire face à la crise.

Les patients rencontrés lors des entretiens soulignent la qualité du soutien émotionnel offert par leurs proches. Nous n'avons pas pris en compte l'ajustement dyadique à la maladie. Pourtant de récents travaux témoignent de l'importance de considérer la maladie chronique et ses stresseurs dans un contexte relationnel (Bard \& Acitelli, 2017). Il conviendrait d'enrichir notre étude en intégrant cette dimension.

De plus, nous pouvons, souligner un biais relatif à l'engagement des patients dans l'étude soutenu par les professionnels de santé engagés eux-mêmes dans la recherche. En outre, nous ne disposons pas d'informations sur la présence d'un suivi psychothérapeutique chez les patients qui aurait pu soutenir le processus dynamique de construction de stratégies de coping spécifiques. 


\section{Conclusion}

Notre étude a permis d'identifier à partir du vécu de la MC, les stratégies de coping centrées sur le problème, l'émotion et la recherche de soutien social. Si certaines sont retrouvées classiquement dans la littérature, la perspective qualitative de l'étude, nous permet de constater qu'elles sont employées en fonction de stresseurs spécifiques, dépendant de l'expérience subjective de la maladie comme la stigmatisation associée aux symptômes, l'impossibilité de maîtriser ceux-ci ou la peur d'une rechute. Ainsi, les futures études, notamment sur le développement des échelles de coping, doivent davantage prendre en compte les stratégies de coping répertoriées en lien avec l'expérience de la MC des patients avec l'exploration de dimensions relatives à la fonction adaptative des émotions positives ainsi qu'à l'émergence de savoirs profanes.

À travers l'investigation de ces stratégies dans une perspective diachronique, c'est-à-dire, traitant du vécu passé et actuel de la maladie, nous avons pu mettre en lumière l'apparition de stratégies centrées sur les savoirs expérientiels des patients et sur la fonction adaptative des émotions positives. Ces deux stratégies nous ouvrent des perspectives supplémentaires pour penser l'accompagnement psychologique des patients. Il convient d'identifier à travers le récit de l'expérience de la maladie, la mise en place de stratégies de coping personnelles fondées sur leur propre expérience et de favoriser la verbalisation des émotions positives. Des lors, il appartient pour le psychologue de veiller à développer le processus narratif du vécu passé et actuel de leur maladie afin de pouvoir faire émerger ces stratégies d'adaptation auprès des patients eux-mêmes. Ces stratégies pourraient alors être conçues comme une opportunité pour développer un sentiment de reprise de contrôle face à une maladie imprévisible et permettre la reconnaissance auprès des professionnels de santé d'une expertise profane de la MC.

\section{Déclaration de liens d'intérêts}

Les auteurs déclarent ne pas avoir de liens d'intérêts.

\section{Remerciements}

Cette recherche a été soutenue par l'Association François Aupetit (AFA). Nous remercions l'ensemble des patients ayant participé à cette recherche.

\section{Références}

Aïni, K., Curelli-Chéreau, A., \& Antoine, P. (2010). L’expérience subjective de patients avec une fibromyalgie : analyse qualitative. Annales Médico-psychologiques, 168(4), 255-262.

Bard, H., \& Acitelli, L. K. (2017). Re-thinking dyadic coping in the context of chronic Illness. Current Opinion in Psychology, 13, 44-48.

Bloor, M., \& Wood, F. (2006). Keywords in qualitative methods. A vocabulary of research concepts. Thousand Oaks: Sage.

Braun, V., \& Clarke, V. (2006). Using thematic analysis in psychology. Qualitative Research in Psychology, 3(2), 77-101.

Carver, C. S., Scheier, M. F., \& Weintraub, J. K. (1989). Assessing Coping Strategies: A theoretically based approach. Journal of Personality and Social Psychology, 56, 267-283.

Carver, C. S. (1997). You want to measure coping but protocole's too long: consider the Brief COPE. The International Journal of Behavioral Medicine, 4(1), 92-100.

Casati, J., Toner, B. B., De Rooy, D., Drossman, D. A., \& Maunder, R. (2000). Concerns of patients with Inflammatory Bowel Disease. A review of Emerging Themes. Digestive Diseases and Sciences, 45(1), 26-31.

Bitton, A., Dobkin, P. L., Edwardes, M. D., Sewitch, M. J., Meddings, J. B., Rawal, S., Cohen, A., Vermeire, S., Dufresne, L., Franchimont, D., \& Wild, G. E. (2008). Predicting relapse in Crohn's disease: a biopsychosocial model. Gut, 58, 1386-1392.

Byron, C., Cornally, N., Burton, A., \& Savage, E. (2020). Challenges of living with and managing inflammatory bowel disease: A meta-synthesis of patients' experiences. J Clin Nurs, 29, 305-319.

Cooper, J. M., Collier, J., James, V., \& Hawkey, C. J. (2010). Beliefs about personal control and self-management in 30-40 years olds living with inflammatory bowel disease: a qualitative study. International journal of nursing studies, 47, 1500-1509.

De Ridder, D., Geenen, R., Kuijer, R., \& Van Middendorp, H. (2008). Psychological adjustement to chronic disease. Lancet, 372, $246-255$.

Endler, N. S., \& Parker, J. D. A. (1990). Coping Inventory for Stressful Situations (CISS): manual. Toronto, Canada: Multi-Health Systems.

Folkman, S., Chesney, M. A., Collette, L., Boccellari, A., \& Cooke, M. (1996). Postbereavement depressive mood and its prebereavement predictors in HIV+ and HIVgay men. Journal of Personality and Social Psychology, 70, 336-348.

Folkman, S. (2008). The case for positive emotions in the stress process. Anxiety, Stress and Coping, 21(1), 3-14. 


\section{A. Lainé et al.}

Fourie, S., Jackson, D., \& Aveyard, H. (2018). Living with inflammatory Bowel Disease: a review of qualitative research studies. International Journal of Nursing Studies, 87, 149-156.

Francis, J. J., Johnston, M., Robertson, C., Glidewell, L., Entwistle, V., Eccles, M. P., \& Grimshaw, J. M. (2010). 'What is Adequate Sample Size? Operationalising Data Saturation for Theory-Based Interview Studies'. Psychology and Health, 25, 1229-1245.

Graff, L. A., Walker, J. R., Clara, I., Lix, L., Miller, N., Rogala, L., rawsthorne, R. N., Charles, N., \& Bernstein, M. D. (2009). Stress coping, distress, and health perceptions in inflammatory bowel disease and community controls. American Journal of Gastroenterology, 104(12), 2959-2969.

Guest, G., Bunce, A., \& Johnson, L. (2006). How many interviews are enough?: An experiment with data saturation and variability. Field Methods, 18, 59-82.

Hall, N. J., Rubin, G. P., Dougall, A., Hungin, A. P. S., \& Neely, J. (2005). The fight for "Health-related Normality": A qualitative Study of the Experiences of Individuals Living with Established Inflammatory Bowel Disease (IBD). Journal of Health Psychology, 10(3), 443-455.

Irvine, E. J. (2004). Review article: patients' fears and unmet needs in inflammatory bowel disease. Aliment Pharmacology Therapy, 20(4), 54-59.

Lainé, A., Laurent, A., Mariage, A., Boschetti, G., Charlois, A. L., Roblin, X., Bommelaer, Nancey, S., \& Flourié, B. (2016). Facteurs psychosociaux et risque de rechute au cours de la maladie de Crohn. Annales Médico-Psychologiques, 174, 461-467.

Larsson, K., Lööf, L., \& Nordin, K. (2017). Stress, coping and support needs of patients with ulcerative colitis or Crohn's disease: a qualitative descriptive study. Journal of clinical nursing, 26(5-6), 648-657.

Laurent, A., Reignier, J., Le Gouge, A., Azoulay, E., Robert, R., \& Kentish-Barnes, N. (2019). "You helped me keep my head above water" - experience of bereavement research after loss of a loved one in the ICU: insights from the ARREVE study. Intensive Care Medicine, 45(9), 1252-1261.

Laurent, A., Bonnet, M., Capellier, G., Aslanian, P., \& Hebert, P. (2017). Emotional impact of end-of-life decisions on professional relationships in the intensive care unit: an obstacle to collegiality? Critical Care Medecine, 45(12), 2023-2030.

Lazarus, R. S., \& Folkmann, S. (1991). The concept of coping. In A. Monat E R. S. Lazarus (Eds.), Stress and coping: An anthology. Columbia University Press.

Lazarus, R. S., \& Folkmann, S. (1984). Stress Apparaisal, and Coping. New York: Spinger.

Livneh, H. (2019). The use of generic avoidant coping scales for psychological adaptation to chronic illness and disability: a systematic review. Health psychology Open, 6(2), 1-17.

Luo, H., Sun, Y., Li, Y., Lv, H., Sheng, L., Wang, L., \& Qian, J. (2018). Perceived stress and inappropriate coping behaviors associated with poorer quality of life and prognosis in patients with Ulcerative colitis. Journal of psychosomatic Research, 113, 66-71.

Maes, S., Leventhal, H., \& De Ridder, D. T. D. (1996). Coping with chronic disease. In M. Zeidner, \& N. S. Endler (Eds.), Handbook of coping. Theory, Research, Applications. New York: Wiley (pp. 221-251).

Mc Combie, A. M., Mulder, R. T., \& Gearry, R. B. (2013). How IBD patients cope with IBD: a systematic review. Journal of Crohn's and Colitis, 7(2), 89-106.

McCormick, J. B., Hammer, R. R., Farrell, R. M., Geller, G., James, K. M., Loftus, E. V., \& Sharp, R. R. (2012). Experiences of patients with chronic gastrointestinal conditions: in their own words. Health and Quality of Life Outcomes, 10, 25-36.

Pelissier, S., Dantzer, C., Canini, F., Mathieu, N., \& Bonaz, B. (2010). Psychological adjustment and autonomic disturbances in inflammatory bowel diseases and irritable bowel syndrome. Psychoneuroendocrinology, 35(5), 653-662.

Schwarzer, R., \& Renner, B. (2000). Social-cognitive predictors of health behavior: action self-efficacy and coping self-efficacy. Health Psychology, 19, 487-495.

Schwarzer, R., \& Knoll, N. (2002). Positive Coping: Mastering Demands and Searching for Meaning. To appear. In S. J. Lopez, \& C. R. Snyder (Eds.), Handbook of Positive Psychological Assessment. Washington, DC: American Psychological Association.

Stroebe, M., Schut, H., \& Stroebe, W. (2005). Attachment in coping with bereavement: a theoretical integration. Review of General Psychology, 9(1), 48-66.

Taft, T. H., Keefer, L., Artz, C., Bratten, J., \& Jones, M. P. (2011). Percptions of illness stigma in patients with inflammatory bowel disease and irritable bowel syndrome. Quality of Life Research, 20, 1391-1399.

Van der Have, M., Minderhoud, I. M., Kaptein, A. A., Leenders, M., Siersema, P. D., Fidder, H. H., \& Oldenburg, B. (2013). Substantial impact of illness perceptions on quality of life in patients with Crohn's disease. Journal of Crohn's and Colitis, 7(8), e292-e301.

Vitaliano, P. P., Russo, J., Carr, J., \& Maiuro, R. (1985). The Ways of Coping Checklist: Revision and Psychometric Properties. Multivariate Behavioral research, 20(1), 3-26. 